\title{
GENERALIZATIONS OF THE THEOREM OF FERMAT AND CAUCHY ON POLYGONAL NUMBERS*
}

BY L. E. DICKSON

1. Introduction and Summary. We seek the least $l$ such that every integer $A \geqq 0$ is a sum of $l$ values of

$$
p_{m+2}(x-k)=\frac{1}{2}(x-k)[m(x-k-1)+2]
$$

for integers $x \geqq 0$. When $k=0$, (1) is a polygonal number of order $m+2$, and Fermat stated that $l=m+2$. This was first proved by Cauchy, who found that all but four of the $m+2$ polygonal numbers may be taken as 0 or 1 . A simpler proof was given by the writer in this Bulletin (vol 33 (1927), pp. 719-726) that paper will be cited as I.

When all but four of the $l$ values of (1) are 0 or 1 , we shall prove here that

$l=m-1$ if $k=1, m \geqq 7 ; l=m-2$ if $k=2, m \geqq 8$, or if $k \geqq 3, m \geqq 7 ; l=6$ if $k=1, m=6$, or $k=2, m=7 ; l=5$ if $k=1, m=4$ or 5 , or if $k \geqq 2, m=6 ; l=4$ if $k \geqq 1, m=3$, or if $k \geqq 2, m=4$ or 5 .

When all but $s$ of the $l$ values of (1) are 0 or 1 , where $5 \leqq s \leqq l$, we shall prove that, for every $k \geqq 1$,

$$
l=m-2 \text { if } m>7, l=s \text { if } m \leqq 7 .
$$

But $A$ is a sum of $l$ values of (1) if and only if $\dagger$

$$
8 m A+l(m-2)^{2}=\sum_{l}(2 m x+2-m-2 m k)^{2},
$$

summed for $l$ integers $x \geqq 0$. We saw that $l=4$ when $k=1$, $m=3$, and when $k=2, m=4$ or 5 . Hence (2) shows that for every integer $A \geqq 0$,

* Presented to the Society, September 9, 1927.

$\dagger$ This implies the like formula with $k$ replaced by any larger integer $k+p$. The values $x \geqq p$ give the same squares as occur in (2) for $x \geqq 0$. Hence we use the least permissible $k$. 
$24 A+4=\sum_{4}(6 x-7)^{2}, \quad 40 A+36=\sum_{4}(10 x-23)^{2}$,

$$
8 A+4=\sum_{4}(4 x-9)^{2} .
$$

By Theorem 6 and (2), we have

$$
3 A+4=\sum_{4}(3 x-16)^{2} \quad \text { if } A \not \equiv 4 \quad(\bmod 8) .
$$

For $s=5, m \leqq 7$, we saw that $l=5$. Then (2) for $k=1$ is

$$
8 m A+5(m-2)^{2}=\sum_{5}(2 m x+2-3 m)^{2} .
$$

For $m=3$ this result is obtained by adding 1 to each member of $\left(3_{1}\right)$ and is discarded. When $m$ is 4 or 6 , we cancel factors 4 or 16 . We get

(6) $8 A+5=\sum_{5}(4 x-5)^{2}, \quad 40 A+45=\sum_{5}(10 x-13)^{2}$,

(7) $3 A+5=\sum_{5}(3 x-4)^{2}, 56 A+125=\sum_{5}(14 x-19)^{2}$.

The results obtained when $s=4, l=5$ are consequences of these.

When $k=1, m \geqq 8$, Theorem 2 states that $l=m-2$ except for $A=54 m+16$. This is a remarkable fact since the single exceptional value may be made as large as we please by taking $m$ sufficiently large. Again, if $k=5, m=6$, we find that $l=4$ fails first for $A=980$; by increasing $k$, the first failure will occur for a value of $A$ exceeding any assigned number.

In later papers I shall prove that the value of $l$ obtained when $s=3$ is never less than the minimum $l$ found here, and I shall give an improved method valid when $s>4$, but not when $s=4$.

2. Formulas. Give (1) the notation $\frac{1}{2} m x^{2}+\frac{1}{2} n x+c$. Thus

$$
n=2-m-2 m k, \quad c=\frac{1}{2} m\left(k^{2}+k\right)-k .
$$

For $k \geqq 1$ the reduced minor conditions in I now follow from

$$
A \geqq 4 c+4 E, \quad A \geqq 4 c+\frac{2}{3} m .
$$


For, from the inequality $\left(9_{2}\right)$ we deduce (11) of I, since the sum $(-n)(2 m+n) / m$ of its last two terms is negative, because $-n$ is positive and $2 m+n$ is negative. By $\left(8_{1}\right)$, we may write (4) of $I$ in the form

$$
A=m g+4 c+b+r, \quad g=\frac{1}{2}(a-b-2 k b) \text {. }
$$

3. Excess $E$ when $k=1$. The values of (1) for $x \geqq 0$ are $m-1$ and all the polygonal numbers of order $m+2$.

TABLE II.

Sums by Four of $m-1$ and Polygonal Numbers

$0-4, m-1, m, m+1-5,2 m-2,2 m-1,2 m, 2 m+1-6,3 m-3,3 m-2$, $3 m, 3 m+1,3-7,4 m-4,4 m-1,4 m+2-8,5 m+1,2,4,5,7,8,6 m, 6 m+3-9$, $7 m+3-9,8 m+2-10,9 m+1,4,7-10,10 m+5-11,11 m+4-9,11,12 m+3$; $4,6-12,13 m+2,5,7-12,14 m+6-12,15 m+6-9,11-13,16 m+5-13$, $17 m+4,5,7-13,18 m+3,6,7,9-14,19 m+8,9,11-14,20 m+7,10-14$, $21 m+7-13,15,22 m+6-15,23 m+5,6,8,9,11-15,24 m+4,7,10-16$, $25 m+9-13,15,16,26 m+8,10,11,13-16,27 m+9,11-16,28 m+8-17$, $29 m+7-13,15-17,30 m+6,7,9,10,12-17,31 m+5,8,11-17,32 m+10-18$, $33 m+9,10,12,13,15-18,34 m+12-18,35 m+11-17,36 m+9-19,37 m$ $+8-13,15-19,38 m+7,8,10,11,13-15,17-19,39 m+6,9,12-17,19$, $40 m+11,12,14-20,41 m+10,13,15-20,42 m+13-20,43 m+12-17,19,20$, $44 m+11-20,45 m+10-13,15-21,46 m+9-21,47 m+8,9,11-17,19-21$, $48 m+7,10,12-21,49 m+12,13,15-21,50 m+11,14-22,51 m+13-17,19-$ $22,52 m+13-22,53 m+12,13,15-21,54 m+17-19,21,22$.

If $m \geqq 6, E(9 m+6)=2$, since neither $9 m+5$ nor $9 m+6$ is equal to a number of Table II. If $m=5$, Table II lacks $17=2 m+7=3 m+2=4 m-3$. If $m=4$, it lacks $26=4 m+10$ $=5 m+6=6 m+2=7 m-2$. Also, $* E(54 m+16)=m-5$. Hence $E$ is not smaller than the value shown in

Theorem 1. For $k=1, E=m-5$ if $m \geqq 7, E=2$ if $m=6$, $E=1$ if $m=4$ or $5, E=0$ if $m=3$.

By (10) we require that the values of $b+r$ shall include a complete set of residues modulo $m$ when $r$ takes the values $0,1, \cdots, E$, and $b$ takes certain consecutive odd values. When $m=3$ or $m \geqq 5$, this will be true if $b$ takes the values

* And independently of Table II, since the only partitions of 53 into $0,1,3,6,10,15,21,28,36,45$ are $1+1+6+45,1+1+15+36,1+6+10+$ $36,1+3+21+28,0+10+15+28,1+10+21+21$. 
$\beta, \beta+2, \beta+4$, whence $d=6$. But when $m=4$, we need only $\beta, \beta+2$, whence $d=4$. Conditions (9) hold if $A \geqq 8 \mathrm{~m}$.

Let $m \geqq 7$. By (6)-(15) of I,

$$
\begin{aligned}
U= & 24 m A-63 m^{2}+12 m+36, \quad V=2 m A-m^{2}+6 m+4, \\
P=7 m+2, \quad W=3 m A-14 m^{2}-2 m+4, & \\
F=m^{2} A^{2}-74 m^{3} A-28 m^{2} A+193 m^{4}+ & 70 m^{3}-68 m^{2} \\
& -24 m>0 .
\end{aligned}
$$

The last evidently holds if

$$
A \geqq 74 m+28 .
$$

Next, let $m=6$. Then

$$
\begin{array}{r}
U=36(4 A-60), V=12 A-8, P=44, W=18 A-512, \\
F=36\left[(7 A-88)^{2}-(12 A-8)(4 A-60)\right] \\
=36\left(A^{2}-480 A+7264\right),
\end{array}
$$

and $F>0$ if $A \geqq 465$ and hence if (11) holds.

If $m \geqq 6$, Theorem 1 now follows from Lemma 3 of I and the following lemma.

Lemma 1. If $m \geqq 5$ in Table II, $E(A) \leqq m-5$ when $A$ is between any consecutive blocks, while $E(A) \leqq 2$ when $A$ is in any block.

For, the difference between consecutive entries in any block is always $\leqq 3$. If $r$ is the term free of $m$ in the leader $q m+r$ of any block, then $r+4$ is found to be the term free of $m$ of a number of Table II within the preceding block. Hence $q m+r-1$ is the sum of $m-5$ and the number $(q-1) m+r+4$ in the table.

When $m=5$, the last two sentences hold after we suppress from Table II all entries down to and including the last entry which differs by 3 from the next entry of the block. The only exception is the leader $9 m+7$, while $9 m+6$ exceeds $8 m+10$ by 1 . Hence $E(A) \leqq 1$ if $A \leqq 54 m+22$.

$$
\begin{gathered}
\text { For } m=5, d=6, n=-13, c=4, \\
U=120 A-1479, V=10 A-1, P=37, W=15 A-356, \\
F=25 A^{2}-10150 A+126685>0 \text { if } A>393=74 m+23 .
\end{gathered}
$$


These facts with Lemma 3 of I prove Theorem 1 for $m=5$.

$$
\begin{gathered}
\text { For } m=4, d=4, n=-10, c=3, \\
U=4(24 A-231), V=4(2 A-1), P=14, W=12 A-140, \\
F=16\left[(7 A-37)^{2}+(2 A-1)(24 A-231)\right] \\
=16(A-16)^{2}+16 \cdot 882>0 .
\end{gathered}
$$

Finally, let $m=3$. Then $d=6, n=-7, c=2$,

$$
\begin{array}{r}
U=9(8 A-55), V=6 A+1, P=23, W=9 A-128, \\
F=9\left[(7 A-42)^{2}-(6 A+1)(8 A-55)\right] \\
=9\left(A^{2}-266 A+1819\right)>0,
\end{array}
$$

if $A \geqq 259$. For $A<259$, Theorem 1 was verified by Tables I and II.

4. Theorem 2. If $k=1, m \geqq 8, E(A) \leqq m-6$ except for $A=54 m+16$.

Within every block of Table II the difference of any two consecutive entries is $\leqq 3$. If $f$ is the term free of $m$ in the leader $q m+f$ of any block having $q \neq 16,46,52,54$, then $f+5$ is that of a number $t$ occurring explicitly in the table. Hence $q m+f-1$ is the sum of $m-6$ and $t=(q-1) m+f+5$. For $q=16,46$, and 52 , a like result holds if we replace $f+5$ by $f+6$ and hence replace $m-6$ by $m-7$. Hence the theorem is true for $A<54 m+16$. For $54 m+17 \leqq A \leqq 199 m$ +37 , it is true by Lemmas 3 and 4 of I.

For $A \geqq 199 m+38, E=m-6$, we have $d=8$,

$$
\begin{gathered}
U=24 m A-63 m^{2}+12 m+36, \quad V=2 m A-m^{2}+8 m+4 \\
P=11 m+2, \quad W=3 m A-23 m^{2}-4 m+4 \\
F=m^{2} A^{2}-200 m^{3} A-48 m^{2} A+562 m^{4}-84 m^{3} \\
-264 m^{2}-48 m .
\end{gathered}
$$

Then $F>0$, in fact for $A=198 m+44+t, t \geqq 0$. 
5. Excess $E_{s}$ when $k=1$.

Theorem 3. When $k=1, E_{s}=0$ if $m \leqq 7, s \geqq 5$, and if $m>7, s \geqq m-2$, while $E_{s}=m-s-2$ if $m>7,5 \leqq s \leqq m-2$.

If $m \leqq 5$, this follows from Theorem 1 . If $m>7, E_{5} \leqq m-7$ by Theorem 2 and the fact that $54 m+16$ is the sum of the polygonal number $3 m+3$ and the entry $51 m+13$ of Table II. Since the polygonal numbers $>1$ exceed $m-1$, the summands yielding $m-2$ are all 1 , whence $E_{s}(m-2)=m-2-s$. Hence $E_{5}=m-7$. If $s>5$, regard $s-5$ of the values 1 as polygonal numbers; hence $E_{s} \leqq m-7-(s-5)$. This proves Theorem 3 except for $m=6$ and 7 .

For $m=6$ or 7 , we seek a constant $C_{m}$ such that $E_{4}(A) \leqq 1$ for every $A \geqq C_{m}$. When $r$ takes the values 0 and $E=1$, and $b$ takes 3 or 4 consecutive odd values according as $m=6$ or $m=7$, the values of $b+r$ include a complete set of residues modulo $m$, whence $d=6$ or 8 , respectively.

In the discussion in $\S 3$ for $m \geqq 7$, we had $d=6, E=m-5$. Hence it is valid here for $m=6, d=6, E=1$, and shows that $E_{4}(A) \leqq 1$ if $A \geqq 74 m+28$. Likewise, the work at the end of $\S 4$ is valid here for $m=7, d=8, E=1$, and shows that $E_{4}(A) \leqq 1$ if $A \geqq 198 m+44$.

It remains to treat the values of $A$ below these two limits. From Table II we readily deduce a list of the sums by five of $m-1$ and polygonal numbers and then verify for $m=6$ and $m=7$ that the list includes all positive integers $\leqq 54 m+25$. From thence to $74 m+28, E_{4}(A) \leqq 1$ if $m=6$ or 7 by Lemma 3 of I. If $m=7$ and $74 m+20 \leqq A \leqq 199 m+37$, $E_{4}(A) \leqq 1$ by Lemma 4 of $\mathrm{I}$. This completes the proof of Theorem 3.

6. Excess $E$ when $k=2$. The values of (1) for $k=2, x \geqq 0$ are $m-1,3 m-2$, and all the polygonal numbers of order $m+2$. Evidently $E(m-2)=m-6$. For $m=7$ the sums by four of the values mentioned were tabulated to 395 ; the only consecutive integers missing from the list are 393-4; hence $E(394)=2, E(A) \leqq 1$ if $A<394$. For $m=6, E(60)=1$. Hence $E$ is not smaller than the value shown in 
Theorem 4. For $k=2, E=m-6$ if $m \geqq 8, E=2$ if $m=7$, $E=1$ if $m=6, E=0$ if $m=3,4$, or 5 . If $m=7, E(A) \leqq 1$ except for $A=394$.

Conditions (9) are satisfied if $A \geqq 16 m$. For $r=0,1, \cdots$, $E$, we require that $b+r$ shall include a complete set of residues modulo $m$. When $m \geqq 8$, this will be true if $b$ takes the values $\beta, \beta+2, \beta+4, \beta+6$; when $m=5$, also $\beta+8$; when $m=6$ or 7 , only the first three.

First, let $m \geqq 8$. Then $d=8$,

$$
\begin{array}{r}
U=24 m A-135 m^{2}+36 m+36, \quad P=9 m+2, \\
V=2 m A-m^{2}+8 m+4, \quad W=3 m A-27 m^{2}+4, \\
F=m^{2} A^{2}-112 m^{3} A-40 m^{2} A+706 m^{4}+188 m^{3}-152 m^{2} \\
-48 m .
\end{array}
$$

Evidently $F>0$ if $A \geqq 112 m+40$. For smaller $A$ 's exceeding $54 m+16$, Lemmas 3 and 4 of I show that $E(A) \leqq m-6$ if $m \geqq 7$.

Lemma 2. If $m \geqq 7$ in Table II, $E(A) \leqq m-6$ except for $A=9 m+6,20 m+9$, and $54 m+16$.

From each block we suppress all entries down to and including the last entry which differs by 3 from the next entry. Proceed as in $\S 4$. We now have the further exceptions $q=9$ and 20. Also

$$
\begin{aligned}
9 m+3 & =m-6+t_{1}, \quad 9 m+5=1+t_{2}, \\
20 m+8 & =m-6+t_{3}, \quad 54 m+15=m-6+t_{4},
\end{aligned}
$$

where the $t_{i}$ occur in the table.

For $m \geqq 8$, Theorem 4 now follows since

$$
\begin{gathered}
9 m+6=1+2(3 m+3)+3 m-2+1,20 m+9=m+2 \\
+6 m+4+10 m+5+3 m-2, \\
54 m+16=2(3 m+3)+45 m+10+3 m-2+2 .
\end{gathered}
$$

For $m=7, E=2, d=6, P=37$,

$$
\begin{aligned}
& U=168 A-6327, \quad V=14 A-3, \quad W=21 A-962, \\
& F / 7=7 A^{2}-826 A+131149,
\end{aligned}
$$


and $F>0$ for every $A>0$. But if we attempt to use $E=1$, we have $d=8$; the discussion for $m \geqq 8$ now applies also for $m=7$ except when $A=54 m+16=394$. This proves both parts of Theorem 4 if $m=7$.

Next, let $m=6$. Then $E=1, d=6, P=32$,

$$
\begin{gathered}
U=9(16 A-512), \quad V=4(3 A+1), \quad W=18 A-704 \\
F / 36=(7 A-116)^{2}-(3 A+1)(16 A-512)=(A-52)^{2} \\
+11264 .
\end{gathered}
$$

The minor conditions in $I$ are all satisfied if $A \geqq 44$. For $m=6$, Table II includes all numbers less than 44 except $33=1+2(3 m-2)$.

Let $m=5$. Then $E=0, d=10, P=67$,

$$
\begin{aligned}
& U=120 A-3159, \quad V=10 A+9, \quad W=15 A-956, \\
& F / 25=(A-703)^{2}-457878>0 \text { if } A \geqq 1380 .
\end{aligned}
$$

It was verified that every integer $\leqq 1380$ is a sum by four of 4,13 , and polygonal numbers of order 7 , whence $E=0$.

Let $m=4$. Then $n=-18, c=10, A=2 a-9 b+40$. Our general method requires that $a$ and $b$ be odd and applies only when $A$ is odd. By $(10)$ with $r=0, b \equiv A(\bmod 4)$. Hence $d=4, P=6$,

$$
\begin{gathered}
U=4(24 A-495), \quad V=4(2 A+1), \quad W=4(3 A-63), \\
F / 16=(7 A-61)^{2}-(2 A+1)(24 A-495)=A^{2}+112 A \\
+4216,
\end{gathered}
$$

whence $F>0$ for every $A \geqq 0$. The minor conditions in I are all satisfied if $A \geqq 28$. For $m=4$, Table II includes all integers $\leqq 28$ except $26=m+2+2(3 m-2)$.

Next, let $A$ be even. Since $b$ must be even, our previous method is not applicable.

Lemma $3 .^{*}$ If $b=2 B, a$ is even, $a-B^{2}$ is a sum of three squares, and

* Legendre, Theorie des Nombres, ed. 3, II, No. 628, with omission of denominator 2 in No. 629. 


$$
4 B^{2}+2 B+1>3 a,
$$

then $a=\sum \alpha^{2}, b=\sum \alpha$ have solutions $\alpha, \beta, \gamma, \delta$ in integers $\geqq 0$.

Write $A=2 S$. Then $a=S+9 B-20$. Inserting this value of $a$ into (12) and multiplying by 16 , we see that (12) holds if

$$
8 B>25+u^{1 / 2}, \quad u=48 S-351 \geqq 0 .
$$

Similarly, $B^{2}<a$ if

$$
2 B<9+v^{1 / 2}, \quad v=4 S+1 \text {. }
$$

Take $B \equiv 2-S(\bmod 4)$. Then $a \equiv 2(\bmod 4), a-B^{2} \equiv 1$ or $2(\bmod 4)$, according as $B$ is odd or even. Hence $a-B^{2}$ is a sum of three squares, and all conditions in Lemma 3 are satisfied.

To insure the choice of $B$ as a prescribed residue modulo 4, we require that the difference between the limits for $B$, determined by (13) and (14), shall exceed 4 . The resulting inequality reduces as usual to

$$
S^{2}-340 S+2440>0, \quad S \geqq 333 .
$$

It remains only to verify Theorem 4 for $m=4$ when $A$ is even and <666. This was done above when $A \leqq 28$. Table II includes all integers from 28 to 238 except 236; Table I includes all from 235 to 666.

7. Excess when $k \geqq 3$. Evidently $E(m-2)=m-6$. If $m=7$,

$$
394=54 m+16=6 m-3+2(10 m+5)+28 m+8+1 .
$$

Hence Theorem 4 implies the cases $m \geqq 7$ of

Theorem 5. For $k \geqq 3, E=m-6$ if $m \geqq 7, E=1$ if $m=6$.

That $E=1$ if $m=6$ follows from Theorem 4 and a result to be proved in a later paper on extended polygonal numbers. It was verified that 116 (or 980) is the least positive integer which is not a sum by four of the values of (1) for $x \geqq 0$, $k=4$ (or $k=5$ ). For $x \geqq 0$, the values $>1$ of (1) are all $\leqq m-1$. Hence the proof in $\S 5$ shows that Theorem 3 holds also when $k>1$. 
8. Theorem 6. If $m=6, k=5, E(A)=0$ except for $A \equiv 4$ $(\bmod 8)$.

If $A$ is odd, our general method applies with

$$
d=6, U=144(A-119), V=12 A+16, P=-4 \text {. }
$$

Then (14) and hence (13) of I holds. Also, (9) hold if $A \leqq 344$, which is below the limit 980 for which $E=0(\S 7)$.

Next, let $A \equiv 2(\bmod 4)$. Take $b=2 B$ and determine $B$ modulo 3 to make $a$ an integer. Then $a \equiv 2(\bmod 4)$. Apply Lemma 3. Then (12) holds and $B^{2}<a$ if

$$
\begin{aligned}
4 B>31+u^{1 / 2}, \quad 3 B & <32+v^{1 / 2}, \quad u=4 A-403, \\
v & =3 A+4 .
\end{aligned}
$$

The difference between these limits for $B$ exceeds $d$ if

$$
4 v^{1 / 2}-3 u^{1 / 2}>R=12 d-35 .
$$

Here $d=3$ and (15) holds for every $A$ such that $u>0$.

Finally, let $A \equiv 0(\bmod 8)$. Take an odd $B$ such that $a$ is an integer, whence $d=6$. Then $a \equiv 4(\bmod 8)$ and Lemma 3 applies. The square of (15) holds if

$$
(A-491)^{2}>65712, \quad A \geqq 748 .
$$

The University of Chicago 